\title{
The influence function for self-equilibrating forces on a semi-infinite wedge
}

\author{
H Qiu, D A Hills*, and D Dini \\ Department of Engineering Science, University of Oxford, Oxford, UK
}

The manuscript was received on 3 July 2006 and was accepted after revision for publication on 22 January 2007.

DOI: 10.1243/03093247JSA249

\begin{abstract}
A solution is derived for the surface displacements arising on the faces of a semiinfinite sharp notch subjected to loading by two equal normal forces, one on each wedge face, applied an equal distance from the notch root. This is achieved by a novel, but robust, procedure using a line of strain nuclei applied along an inclined line within a half-plane and permits good convergence of the solution both in the neighbourhood of the points of application of the forces and at the notch root.
\end{abstract}

Keywords: influence function, self-equilibrating forces, semi-infinite wedge

\section{INTRODUCTION}

This paper is concerned with the determination of the normal displacement for a pair of opposed forces applied to the surfaces of a semi-infinite wedge (Fig. 1). The reason for studying this problem is to provide an influence function permitting the effect of notch closure under remote compression to be studied $[\mathbf{1}]$, although it has application to other notch-contact problems. One possible technique for examining the problem at hand would be to use the Mellin transform [2]. However, the principal feature required in the solution is the surface displacement, and it seems natural to ensure that, if the observation point is close to the force itself, the half-plane solution is recovered. It was also noted that, along the notch bisector $\theta=0$, the shearing stress must vanish, together with the $\theta$-direction displacement. Thus, by imposing along this line the hybrid tractiondisplacement boundary conditions described, the correct boundary conditions along the notch bisector can be achieved, and the half-plane solution automatically recovered when the observation point moves from the 'notch' apex towards the point of application of the force (and this observation applies a fortiori if the observation point is exterior to the line force). The accepted way of achieving a traction

\footnotetext{
* Corresponding author: Department of Engineering Science, University of Oxford, Parks Road, Oxford, OX1 3PJ, UK. email: david.hills@eng.ox.ac.uk
}

boundary condition along an internal line is to employ a distribution of dislocations as strain nuclei [3]. This has been carried out many times and does not merit a detailed description here. On the other hand, point forces are more suited to the imposition of internal displacement boundary conditions, and the development of this parallel technique was undertaken by Dundurs [4] and Markenscoff et al. [5] applied to what they termed 'anticracks'. Although a combination of the crack and anticrack techniques should work in principle, it remained to be seen whether the Cauchy nature of the two kernels, when expressing the state of stress, would be complementary or produce a conflict in the ensuing quadrature. The subject of this paper is therefore twofold. First, it permits the imposition of mixed boundary conditions along an internal line in a plane problem. In this case the line exists within a halfplane, but the problem could equally be applied to other domains in which the solution for nuclei are known. Second, the specific application analysed permits useful influence functions for the problem shown in Fig. 1 to be found.

\section{FORMULATION}

The problem to be addressed is shown in detail in Fig. 1(a). Symmetry conditions show that, along the notch bisector, $u_{\theta}=\tau_{r \theta}=0 \forall r$. It follows that the state of stress in the sector $0 \leqslant \theta \leqslant \alpha$ can be found from 


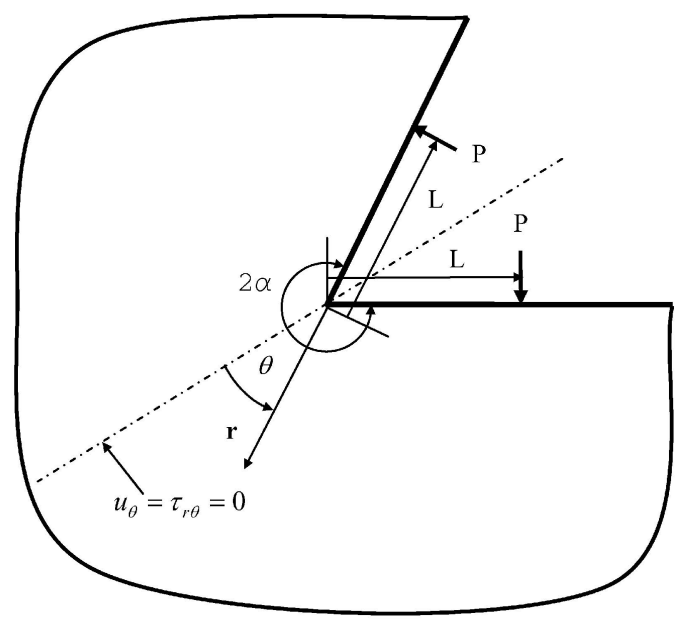

(a)

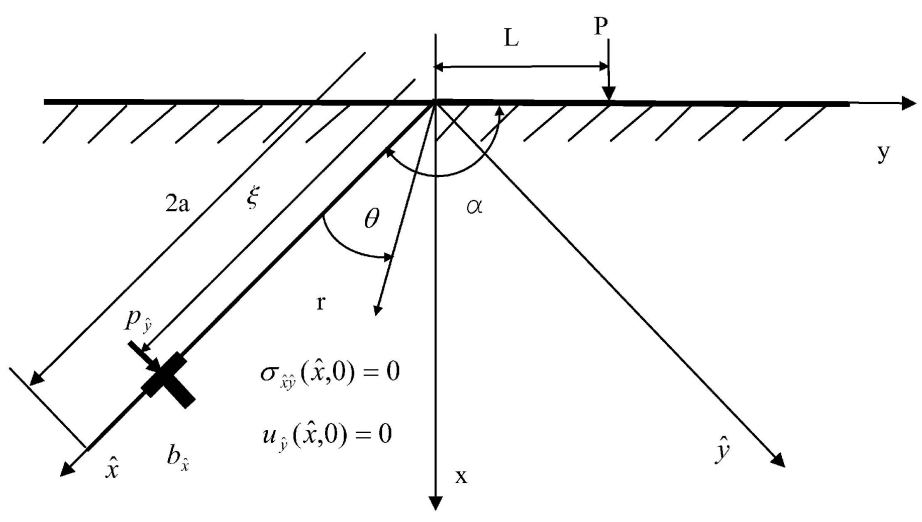

(b)

Fig. 1 (a) A semi-infinite notch under the action of normal forces $P$. (b) A half-plane subject to displacement and traction boundary conditions along the line $\theta=0$, to represent conditions along the notch bisector

the half-plane problem depicted in Fig. 1(b). In order to do this it is required to find the state of stress induced in the half-plane by the normal load $P$ with a correction to establish the condition $u_{\hat{y}}=\tau_{\hat{x} \hat{y}}=0$ on the line $\hat{y}=0, \hat{x} \geqslant 0$. It is assumed that the influence functions giving the state of stress and displacement within a half-plane, due to both a line force and a dislocation, are known. Thus, we know the four influence functions connecting dislocation $b_{k}$ and line force $P_{k}$ with displacement $u_{i}(x, y)$ and state of stress $\sigma_{i j}(x, y)$ (i.e. the functions $G_{k i j}, U_{k i}[\mathbf{3}], S_{k i j}$, and $D_{k i}$; see Appendix 2) defined in the four equations, where $(c, d)$ are the coordinates of the strain nucleus and $(x, y)$ the observation point,

$$
\begin{array}{r}
\sigma_{b i j}(x, y)=\frac{2 \mu}{\pi(\kappa+1)} b_{k}(c, d) G_{k i j}(x, y, c, d), \\
k, i, j=x, y
\end{array}
$$

$$
\begin{gathered}
u_{b i}(x, y)=\frac{1}{2 \pi(\kappa+1)} b_{k}(c, d) U_{k i}(x, y, c, d), \\
k, i=x, y \\
\sigma_{P i j}(x, y)=\frac{1}{2 \pi(\kappa+1)} P_{k}(c, d) S_{k i j}(x, y, c, d), \\
k, i, j=x, y \\
u_{P i}(x, y)=\frac{1}{2 \mu 2 \pi(\kappa+1)} P_{k}(c, d) D_{k i}(x, y, c, d), \\
k, i=x, y
\end{gathered}
$$

Here, $\mu$ is the modulus of rigidity and $\kappa$ is Kolosov's constant.

The intention is to write down two (coupled) singular integral equations to establish the boundary 
conditions along the crack-anticrack line according to

$$
\begin{array}{r}
0=\sigma_{\hat{x} \hat{y}}(\hat{x}, 0) \equiv \bar{\sigma}_{\hat{x} \hat{y}}(\hat{x}, 0)+\tilde{\sigma}_{b \hat{x} \hat{y}}(\hat{x}, 0)+\tilde{\sigma}_{P \hat{x} \hat{y}}(\hat{x}, 0), \\
\hat{y}=0, \quad \hat{x} \geqslant 0 \\
0=u_{\hat{y}}(\hat{x}, 0) \equiv \bar{u}_{\hat{y}}(\hat{x}, 0)+\tilde{u}_{b \hat{y}}(\hat{x}, 0)+\tilde{u}_{P \hat{y}}(\hat{x}, 0), \\
\hat{y}=0, \quad \hat{x} \geqslant 0
\end{array}
$$

where, in each equation, the first term on the righthand side is associated with the surface normal load $P$, while the second is the corrective term associated with dislocations and the third the corrective term associated with point forces, the last two being distributed along the line segment $\hat{y}=0, \hat{x} \geqslant 0$.

Before this is possible two steps are needed. First, we need to establish which of the terms in the four influence functions are singular and which are not, so that there are no conflicting requirements in the quadratures employed. Second, the axis set in which the solutions are expressed needs to be reoriented along the local axis set fixed to the crack-anticrack line, so that the boundary conditions can be properly established. The latter will be considered first.

Two steps in the operation are needed. First, the Burgers vector of the dislocation is, of course, a vector quantitiy, and the standard vector rotation matrix may be employed in order to express the Burgers components in the local axis set according to

$$
\left\{\begin{array}{l}
b_{x} \\
b_{y}
\end{array}\right\}=\left\{\begin{array}{cc}
\cos \left(\frac{\pi}{2}-\alpha\right) & -\sin \left(\frac{\pi}{2}-\alpha\right) \\
\sin \left(\frac{\pi}{2}-\alpha\right) & \cos \left(\frac{\pi}{2}-\alpha\right)
\end{array}\right\}\left\{\begin{array}{l}
b_{\hat{x}} \\
b_{\hat{y}}
\end{array}\right\}
$$

Second, in order to transform the state of stress induced the standard second-order transformation must be employed, which, for consistency with the above vector operation, is written as

$$
\begin{aligned}
\left\{\begin{array}{l}
\sigma_{\hat{x} \hat{x}} \\
\sigma_{\hat{y} \hat{y}} \\
\sigma_{\hat{x} \hat{y}}
\end{array}\right\} \\
=\left\{\begin{array}{lll}
\cos ^{2}\left(\frac{\pi}{2}-\alpha\right) & \sin ^{2}\left(\frac{\pi}{2}-\alpha\right) & \sin (\pi-2 \alpha) \\
\sin ^{2}\left(\frac{\pi}{2}-\alpha\right) & \cos ^{2}\left(\frac{\pi}{2}-\alpha\right) & -\sin (\pi-2 \alpha) \\
-\frac{1}{2} \sin (\pi-2 \alpha) & \frac{1}{2} \sin (\pi-2 \alpha) & \cos (\pi-2 \alpha)
\end{array}\right\} \\
\\
\times\left\{\begin{array}{l}
\sigma_{x x} \\
\sigma_{y y} \\
\sigma_{x y}
\end{array}\right\}
\end{aligned}
$$

while the displacement state transforms also as a vector according to

$$
\left\{\begin{array}{l}
u_{\hat{x}} \\
u_{\hat{y}}
\end{array}\right\}=\left\{\begin{array}{cc}
\cos \left(\frac{\pi}{2}-\alpha\right) & \sin \left(\frac{\pi}{2}-\alpha\right) \\
-\sin \left(\frac{\pi}{2}-\alpha\right) & \cos \left(\frac{\pi}{2}-\alpha\right)
\end{array}\right\}\left\{\begin{array}{l}
u_{x} \\
u_{y}
\end{array}\right\}
$$

Applying the transformation equations to the influence functions and the boundary conditions along the line imposed (equations (5) and (6)) gives the integral equations

$$
\begin{aligned}
& 0= \bar{\sigma}_{\hat{x} \hat{y}}(\hat{x}, 0)+\frac{2 \mu}{\pi(\kappa+1)} \int_{0}^{2 a} B_{\hat{x}}(\xi, 0) G_{\hat{x} \hat{x} \hat{y}}(\hat{x}, 0, \xi, 0) \mathrm{d} \xi \\
&+\frac{1}{2 \pi(\kappa+1)} \int_{0}^{2 a} p_{\hat{y}}(\xi, 0) S_{\hat{y} \hat{x} \hat{y}}(\hat{x}, 0, \xi, 0) \mathrm{d} \xi, \\
& \hat{y}=0, \quad 0 \leqslant \hat{x} \leqslant 2 a \quad(10) \\
& 0=\bar{u}_{\hat{y}}(\hat{x}, 0)+\frac{1}{2 \pi(\kappa+1)} \int_{0}^{2 a} B_{\hat{x}}(\xi, 0) U_{\hat{x} \hat{y}}(\hat{x}, 0, \xi, 0) \mathrm{d} \xi \\
&+\frac{1}{2 \mu 2 \pi(\kappa+1)} \int_{0}^{2 a} p_{\hat{y}}(\xi, 0) D_{\hat{y} \hat{y}}(\hat{x}, 0, \xi, 0) \mathrm{d} \xi, \\
& \hat{y}=0, \quad 0 \leqslant \hat{x} \leqslant 2 a \quad(11)
\end{aligned}
$$

where $B_{\hat{x}}(\xi, 0)=\mathrm{d} b_{\hat{x}} / \mathrm{d} \xi$ and $p_{\hat{y}}(\xi, 0)=\mathrm{d} P_{\hat{y}} / \mathrm{d} \xi$.

The kernels arising in equation 10 can be split into bounded parts, where a prime is added below, together with a Cauchy singular term

$$
\begin{aligned}
& G_{\hat{x} \hat{x} \hat{y}}(\hat{x}, 0, \xi, 0)=\frac{1}{\hat{x}-\xi}+G_{\hat{x} \hat{x} \hat{y}}^{\prime}(\hat{x}, 0, \xi, 0) \\
& S_{\hat{y} \hat{x} \hat{y}}(\hat{x}, 0, \xi, 0)=\frac{1-\kappa}{\hat{x}-\xi}+S_{\hat{y} \hat{x} \hat{y}}^{\prime}(\hat{x}, 0, \xi, 0)
\end{aligned}
$$

Similarly, the kernels arising in equation 11 can be split into a bounded part, where a prime is added, together with a logarithmic singular term according to

$$
U_{\hat{x} \hat{y}}(\hat{x}, 0, \xi, 0)=\frac{1-\kappa}{2} \log (x-\xi)^{2}+U_{\hat{x} \hat{y}}^{\prime}(\hat{x}, 0, \xi, 0)
$$

$$
D_{\hat{y} \hat{y}}(\hat{x}, 0, \xi, 0)=-\kappa \log (x-\xi)^{2}+D_{\hat{y} \hat{y}}^{\prime}(\hat{x}, 0, \xi, 0)
$$


When the singular terms are explicitly separated, equations 10 and 11 can therefore be written in the form

$$
\begin{aligned}
&-\bar{\sigma}_{\hat{x} \hat{y}}(\hat{x}, 0)= \frac{2 \mu}{\pi(\kappa+1)} \int_{0}^{2 a} B_{\hat{x}}(\xi, 0) \\
& \times\left[\frac{1}{\hat{x}-\xi}+G_{\hat{x} \hat{x}}^{\prime}(\hat{x}, 0, \xi, 0)\right] \mathrm{d} \xi \\
&+\frac{1}{2 \pi(\kappa+1)} \int_{0}^{2 a} p_{\hat{y}}(\xi, 0) \\
& \times\left[\frac{1-\kappa}{\hat{x}-\xi}+S_{\hat{y} \hat{x} \hat{y}}^{\prime}(\hat{x}, 0, \xi, 0)\right] \mathrm{d} \xi, \\
&-2 \mu \bar{u}_{\hat{y}}(\hat{x}, 0)= \frac{2 \mu}{2 \pi(\kappa+1)} \int_{0}^{2 a} B_{\hat{x}}(\xi, 0) \\
& \times\left[\frac{1-\kappa}{2} \log (x-\xi)^{2}+U_{\hat{x} \hat{y}}^{\prime}(\hat{x}, 0, \xi, 0)\right] \mathrm{d} \xi \\
&+\frac{1}{2 \pi(\kappa+1)} \int_{0}^{2 a} p_{\hat{y}}(\xi, 0) \\
& \times\left[-\kappa \log (x-\xi)^{2}+D_{\hat{y} \hat{y}}^{\prime}(\hat{x}, 0, \xi, 0) \mathrm{d} \xi, \quad(16)\right. \\
& \hat{y}=0, \quad 0 \leqslant \hat{x} \leqslant 2 a \quad(17)
\end{aligned}
$$

In order to solve the integral, the equations should be written in normalized form through the substitutions

$$
\begin{aligned}
& u=\frac{\xi}{a}-1 \\
& v=\frac{\hat{x}}{a}-1
\end{aligned}
$$

The equations then become

$$
\begin{aligned}
-\bar{\sigma}_{\hat{x} \hat{y}}(v, 0)= & \frac{2 \mu}{\pi(\kappa+1)} \int_{-1}^{1} B_{\hat{x}}(u, 0) \\
& \times\left[\frac{1}{v-u}+a G_{\hat{x} \hat{x} \hat{y}}^{\prime}(v, 0, u, 0)\right] \mathrm{d} u \\
& +\frac{1}{2 \pi(\kappa+1)} \int_{-1}^{1} p_{\hat{y}}(u, 0) \\
& \times\left[\frac{1-\kappa}{v-u}+a S_{\hat{y} \hat{x} \hat{y}}^{\prime}(v, 0, u, 0)\right] \mathrm{d} u, \\
& -1 \leqslant v \leqslant 1
\end{aligned}
$$

$$
\begin{aligned}
& -2 \mu \bar{u}_{\hat{y}}(v, 0) \\
& =\frac{2 \mu}{2 \pi(\kappa+1)} \int_{-1}^{1} B_{\hat{x}}(u, 0) \\
& \quad \times\left[\frac{(1-\kappa) \log (v-u)^{2}}{2}+a U_{\hat{x} \hat{y}}^{\prime}(v, 0, u, 0)\right] \mathrm{d} u \\
& \quad+\frac{1}{2 \pi(\kappa+1)} \int_{-1}^{1} p_{\hat{y}}(u, 0) \\
& \quad \times\left[-\kappa \log (v-u)^{2}+a D_{\hat{y} \hat{y}}^{\prime}(v, 0, u, 0)\right] \mathrm{d} u, \\
& \quad-1 \leqslant v \leqslant 1
\end{aligned}
$$

It is known that, at the corner of the notch, the stress state varies as $r^{\lambda-1}$, where $r$ is a radial coordinate from the 'notch' apex, and $\lambda-1=-0.4555$ for a notch $270^{\circ}$, by using Williams' [6] asymptotic approach. This behaviour is approximated by one which is square-root singular in the choice of fundamental function and hence

$$
\begin{aligned}
& B_{\hat{x}}(u)=\phi_{\hat{x}}(u)\left(1-u^{2}\right)^{-1 / 2} \\
& p_{\hat{y}}(u)=\phi_{\hat{y}}(u)\left(1-u^{2}\right)^{-1 / 2}
\end{aligned}
$$

The side conditions are

$$
\begin{aligned}
& \int_{0}^{2 a} B_{\hat{x}}(\xi) \mathrm{d} \xi=\int_{-1}^{1} B_{\hat{x}}(u) \mathrm{d} u=0 \\
& \int_{0}^{2 a} p_{\hat{y}}(\xi) \mathrm{d} \xi=\int_{-1}^{1} p_{\hat{y}}(u) \mathrm{d} u=0
\end{aligned}
$$

and hence the discretized forms of the integral equations are

$$
\begin{aligned}
&-\bar{\sigma}_{\hat{x} \hat{y}}\left(v_{k}\right) \\
&=\sum_{i=1}^{n} W_{i}\left\{\frac{2 \mu}{\kappa+1} \phi_{\hat{x}}\left(u_{i}\right)\left[\frac{1}{v_{k}-u_{i}}+a G_{\hat{x} \hat{x} \hat{y}}^{\prime}\left(v_{k}, u_{i}\right)\right]\right. \\
&+\frac{1}{2(\kappa+1)} \phi_{\hat{y}}\left(u_{i}\right) \\
&\left.\times\left[\frac{1-\kappa}{v_{k}-u_{i}}+a S_{\hat{y} \hat{x} \hat{y}}^{\prime}\left(v_{k}, u_{i}\right)\right]\right\} \\
& k=1, \ldots, n-1
\end{aligned}
$$




$$
\begin{gathered}
\begin{aligned}
-2 \mu \bar{u}_{\hat{y}}\left(v_{k}\right) & \sum_{i=1}^{n} W_{i}\left\{\frac { \mu } { \kappa + 1 } \phi _ { \hat { x } } ( u _ { i } ) \left[\frac{a(1-\kappa) \log \left(a v_{k}-a u_{i}\right)^{2}}{2}\right.\right. \\
& \left.+a U_{\hat{x} \hat{y}}^{\prime}\left(v_{k}, u_{i}\right)\right] \\
& +\frac{1}{2(\kappa+1)} \phi_{\hat{y}}\left(u_{i}\right) \\
& \left.\times\left[-a \kappa \log \left(a v_{k}-a u_{i}\right)^{2}+a D_{\hat{y} \hat{y}}^{\prime}\left(v_{k}, u_{i}\right)\right]\right\}, \\
k=1, \ldots, n-1 & \text { (25) }
\end{aligned} \\
\sum_{i=1}^{n} W_{i} \phi_{\hat{x}}\left(u_{i}\right)=0 \\
\sum_{i=1}^{n} W_{i} \phi_{\hat{y}}\left(u_{i}\right)=0
\end{gathered}
$$

where

$$
\begin{aligned}
& u_{i}=\cos \left(\frac{2 i-1}{2 n} \pi\right), \quad i=1, \ldots, n \\
& v_{k}=\cos \left(\frac{k}{n} \pi\right), \quad k=1, \ldots, n-1 \\
& W_{i}=\frac{\pi}{n}
\end{aligned}
$$

These represent a system of $2 n$ simultaneous equations for the $2 n$ unknowns $\phi_{\hat{x}}\left(u_{i}\right)$ and $\phi_{\hat{y}}\left(u_{i}\right)$. When these values have been found, the stresses and displacements at an arbitrary point can be determined.

Finally, the left-hand side of equations (24) and (25) represents the stresses and displacements resulting from a point force acting on the surface of a half-plane and are given by Timoshenko and Goodier [7] as

$$
\begin{aligned}
& \bar{\sigma}_{x x}=-\frac{2 P x^{3}}{\pi\left[x^{2}+(y-L)^{2}\right]^{2}} \\
& \bar{\sigma}_{y y}=-\frac{2 P x y^{2}}{\pi\left[x^{2}+(y-L)^{2}\right]^{2}} \\
& \bar{\sigma}_{x y}=-\frac{2 P x^{2} y}{\pi\left[x^{2}+(y-L)^{2}\right]^{2}}
\end{aligned}
$$

$$
\begin{aligned}
\bar{u}_{x}= & -\frac{4 P(y-L)^{2}}{8 \pi \mu\left[x^{2}+(y-L)^{2}\right]} \\
& +\frac{+P\left(x^{2}+(y-L)^{2}\right)(1+\kappa) \log \left[x^{2}+(y-L)^{2}\right]}{8 \pi \mu\left[x^{2}+(y-L)^{2}\right]}-B
\end{aligned}
$$

where the constant $B$ is determined by setting $\bar{u}_{x}$ to zero at a point on the surface. In this case, because at the apex of the notch, the displacements are chosen equal to zero, $\bar{u}_{x}(0)=0$.

\section{RESULTS}

It is required to find the effect of the mixed boundary conditions imposed within the half-plane on the normal displacement induced along the line $x=0$ for $y>0$ (Fig. 1(b)) by the normal point force. Because there is only a single length dimension in the problem $(L)$, this can be used to non-dimensionalize the coordinates.

The total normal displacement along the notch surface is found by taking the effect of the applied point force on a half-plane and adding a corrective solution and is written as

$$
u_{\theta}(0, \hat{r})=\tilde{\mu}_{\theta}(0, \hat{r})+\bar{u}_{\theta}(0, \hat{r})
$$

where $\hat{r}=y / L, \bar{u}_{\theta}$ is the displacement caused by the point force in a half-plane, and $\tilde{u}_{\theta}$ is the displacement caused by the nuclei. The corrective influence function $F_{\theta}(\hat{r})$ for the effect of the strain nuclei alone is defined by

$$
F_{\theta}(\hat{r})=\frac{\mu \tilde{u}_{\theta}(0, \hat{r})}{P}
$$

In the remainder of this paper the main focus of attention is on a single angle for the notch, i.e. $2 \alpha=270^{\circ}$ and Poission's ratio $v$ is set to 0.3 . The influence function $F_{\theta}(\hat{r})$ is plotted in Fig. 2(a), and can be expressed as a series representation of the form

$$
F_{\theta}(\hat{r})=\hat{r}^{\lambda}\left(c_{0}+c_{1} \hat{r}^{1 / 2}+c_{2} \hat{r}+c_{3} \hat{r}^{3 / 2}++c_{4} \hat{r}^{2}\right)
$$




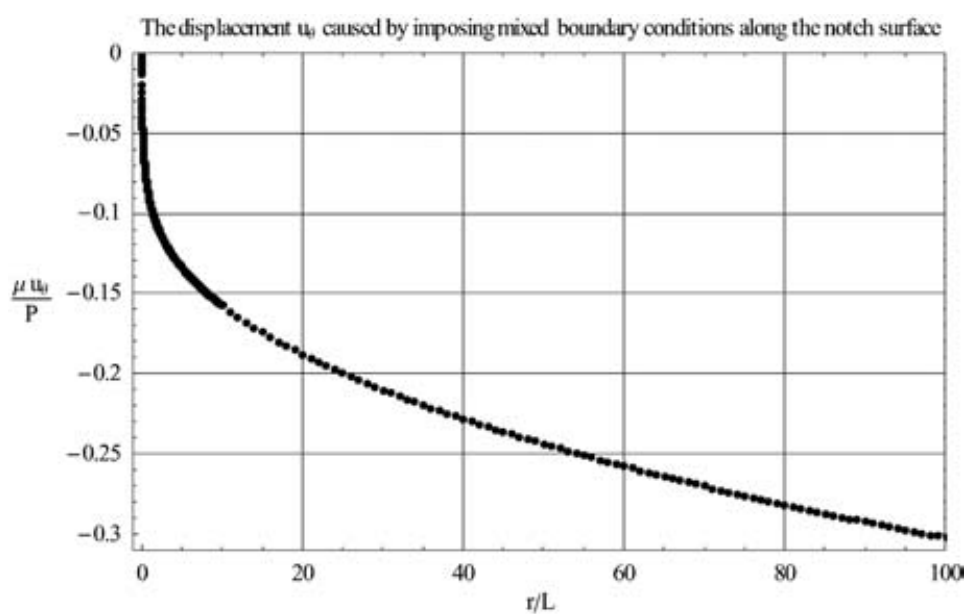

(a)

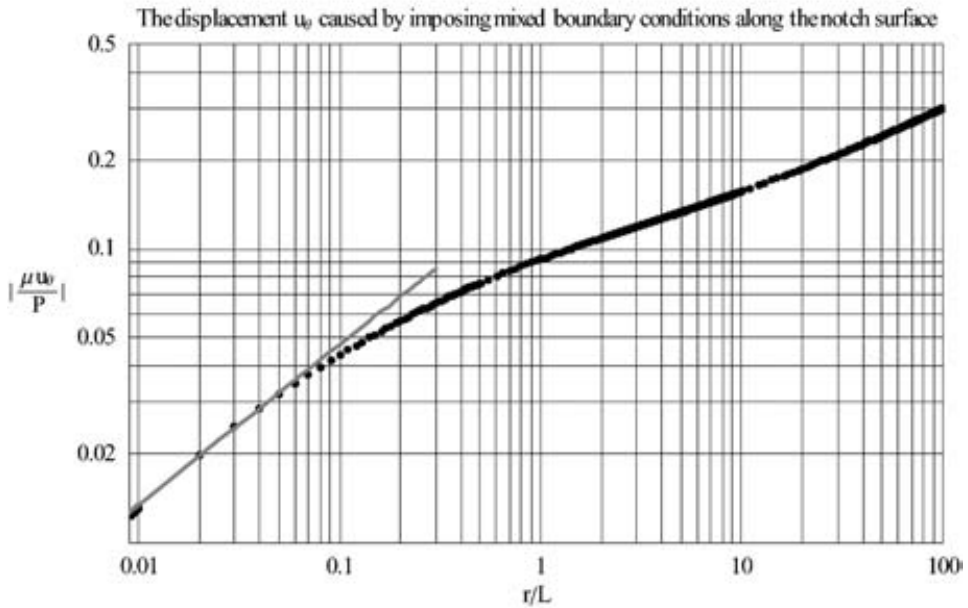

(b)

Fig. 2 The connection displacement generated by the strain nuclei: (a) linear plot; (b) log-log plot of displacement $u_{\theta}$ (full circles) together with the asymptote (grey line)

where $\lambda$ is determined using Williams' [6] asymptotic solution and this gives $\lambda=0.5445$. Figure 2 (b) displays the solution in log-log form, highlighting the characteristic gradients at each end of the range of the solution. When the coefficients have been collected, this gives

$$
\begin{aligned}
F_{\theta}(\hat{r})=\hat{r}^{0.5445}( & -0.1375+0.055 \hat{r}^{1 / 2}-0.0111 \hat{r} \\
& \left.+0.0010 \hat{r}^{3 / 2}-0.00004 \hat{r}^{2}\right)
\end{aligned}
$$

while the displacement caused by the point force is given by

$$
\bar{u}_{\theta}(0, \hat{r})=\frac{P(1-v)}{\mu \pi} \log |\hat{r}-1|
$$

Thus the total normal displacement can be written as

$$
\begin{gathered}
u_{\theta}(0, \hat{r})=\frac{P}{\mu}\left[\hat { r } ^ { 0 . 5 4 4 5 } \left(-0.1375+0.055 \hat{r}^{1 / 2}-0.0111 \hat{r}\right.\right. \\
\left.+0.0010 \hat{r}^{3 / 2}-0.00004 \hat{r}^{2}\right) \\
\left.+\frac{1-v}{\pi} \log |\hat{r}-1|\right]
\end{gathered}
$$

It can be seen that $\hat{r}^{\lambda}$ dominates the $\log |\hat{r}-1|$ term close to the corner of the notch, and $\log |\hat{r}-1|$ dominates the $\hat{r}^{\lambda}$ term in the far field $(\hat{r} / L \geqslant 1)$. The total displacement is plotted in Fig. 3(a) and the value in log-log form is displayed in Fig. 3(b).

In this solution, there are two numerical approximations. One is the truncation of the semi-infinite interval to a finite interval along the inclined line 


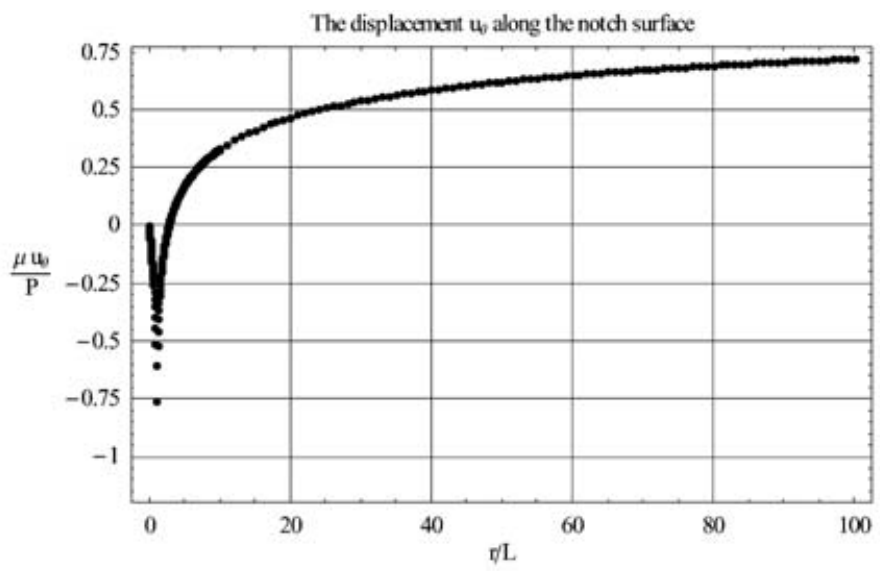

(a)

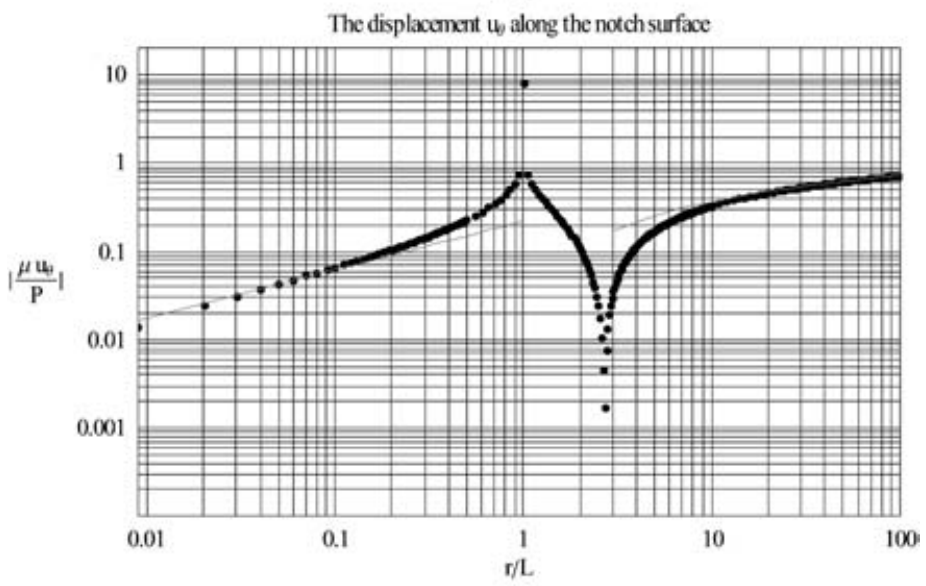

(b)

Fig. 3 State of displacement $u_{\theta}$ along the notch side surface with $\alpha=135^{\circ}$, and $v=0.3$ : (a) linear plot; (b) log-log plot of displacement $u_{\theta}$ (full circles) together with the asymptotes (grey curves)

within the half-plane, and the second is the order $n$ of discretization used in the numerical quadrature. In order to achieve convergence of the solution, setting $a / L$ to 100 was found to be appropriate and, for numerical convergence of the quadrature, it was found that $n=200$ was appropriate.

\section{CONCLUSION}

This paper describes the application of both dislocations and point forces as strain nuclei to achieve mixed boundary value conditions. These have been imposed successfully, and the technique has been used to derive the influence function for a pair of forces applied to the faces on a semi-infinite notch which permits the dominant behaviour adjacent to the notch root, adjacent to the point of application of the force, and remotely to be found.

\section{ACKNOWLEDGEMENTS}

Hui Qiu would like to acknowledge the support of K. C. Wong Foundation and Universities UK for a K. C. Wong Scholarship and an ORS award. David Hills would like to thank the Royal Academy of Engineering for the provision of a Leverhulme Research Fellowship, enabling him to carry out this work.

\section{REFERENCES}

1 Churchman, C. M., Dini, D., and Hills, D. A. Closure at the root of a sharp notch. Int. J. Mech. Sci., 2006, 48, 1063-1071.

2 Barber, J. R. Elasticity, 1992 (Kluwer, Dordrecht).

3 Hills, D. A., Kelly, P. A., Dai, D. N., and Korsunsky, A. M. Solution of crack problems: the distributed dislocation technique, 1996 (Kluwer, Dordrecht). 
4 Dundurs, J. Analogy between concentrated force and edge dislocations. J. Appl. Physics, 1968, 39, 4152-4156.

5 Markenscoff, X., Ni, L., and Dundurs, J. The interface anticrack and Green's functions for interacting anticracks and cracks/anticracks. Trans. ASME, J. Appl. Mechanics, 1994, 61, 797-802.

6 Williams, M. L. Stress singularities resulting from various boundary conditions in angular plates in extension. J. Appl. Mechanics, 1952, 19, 526-528.

7 Timoshenko, S. P. and Goodier, J. N. Theory of elasticity, 3rd edition, 1970 (McGraw-Hill, New York).

\section{APPENDIX 1}

\section{Notation}

$b_{x}, b_{y}$

Burgers vectors of the dislocation

$B_{x}, B_{y}$ density of dislocations

$D_{k i}$

displacement influence function of the line force

$G_{k i j}$ stress influence function of the dislocation

$p_{x}, p_{y}$ density of line forces

$P_{x}, P_{y}$ components of the line force

$S_{k i j}$

$u_{i}$ stress influence function of the line force

$u_{b i}$ displacement component

displacement component due to dislocations

$u_{P i} \quad$ displacement component due to line forces

$\bar{u}_{i} \quad$ displacement component due to external load

$U_{k i} \quad$ displacement influence function of the dislocation

$\sigma_{i j} \quad$ stress component

$\sigma_{b i j} \quad$ stress component due to dislocations

$\sigma_{P i j} \quad$ stress component due to line forces

$\bar{\sigma}_{i j} \quad$ stress component due to the external load

\section{APPENDIX 2}

\section{Influence functions}

$$
\begin{aligned}
S_{x x x}= & \frac{(1-\kappa) x_{1}}{r_{1}^{2}}-\frac{4 x_{1}^{3}}{r_{1}^{4}}+\frac{(\kappa-1)\left(x_{2}-2 c\right)}{r_{2}^{2}} \\
& +\frac{-4 \kappa x_{2}^{3}+(4 \kappa+20) c x_{2}^{3}-24 c^{2} x_{2}}{r_{2}^{4}} \\
& +\frac{32 c^{2} x_{2}^{3}-32 c x_{2}^{4}}{r_{2}^{6}}
\end{aligned}
$$

$$
\begin{aligned}
S_{x y y}= & \frac{(\kappa-5) x_{1}}{r_{1}^{2}}+\frac{4 x_{1}^{3}}{r_{1}^{4}}+\frac{(1-5 \kappa) x_{2}+(2 \kappa+6) c}{r_{2}^{2}} \\
& +\frac{4 \kappa x_{2}^{3}-(4 \kappa+36) c x_{2}^{2}+24 c^{2} x_{2}}{r_{2}^{4}} \\
& +\frac{32 c x_{2}^{4}-32 c^{2} x_{2}^{3}}{r_{2}^{6}}
\end{aligned}
$$

$$
\begin{aligned}
S_{x x y}=(y-d)[ & \frac{1-\kappa}{r_{1}^{2}}-\frac{4 x_{1}^{2}}{r_{1}^{4}}+\frac{\kappa-1}{r_{2}^{2}} \\
& +\frac{-4 \kappa x_{2}^{2}+(12+4 \kappa) c x_{2}-8 c^{2}}{r_{2}^{4}} \\
& \left.+\frac{32 c^{2} x_{2}^{2}-32 c x_{2}^{3}}{r_{2}^{6}}\right]
\end{aligned}
$$

$$
\begin{aligned}
D_{x x}= & {\left[-2 \kappa \log r_{1}+\frac{2 x_{1}^{2}}{r_{1}^{2}}-\left(\kappa^{2}+1\right) \log r_{2}\right.} \\
& \left.+\frac{2 \kappa x_{2}^{2}-4 c x_{2}+4 c^{2}}{r_{2}^{2}}+\frac{8 c x_{2}^{2}\left(x_{2}-c\right)}{r_{2}^{4}}\right]
\end{aligned}
$$$$
D_{x y}=(y-d)\left[\frac{2 x_{1}}{r_{1}^{2}}+\frac{2 \kappa x_{2}-4 \kappa c}{r_{2}^{2}}-\frac{8 c x_{2}\left(c-x_{2}\right)}{r_{2}^{4}}\right]
$$$$
-\left(\kappa^{2}-1\right) \theta_{2}
$$

$$
\begin{aligned}
S_{y x x}=(y-d)\left[\frac{\kappa-1}{r_{1}^{2}}-\frac{4 x_{1}^{2}}{r_{1}^{4}}-\frac{\kappa-1}{r_{2}^{2}}\right. \\
+\frac{-4 \kappa x_{2}^{2}+(4 \kappa-4) c x_{2}+8 c^{2}}{r_{2}^{4}} \\
\left.+\frac{32 c x_{2}^{3}-32 c^{2} x_{2}^{2}}{r_{2}^{6}}\right]
\end{aligned}
$$

$$
\begin{aligned}
S_{y y y}=(y-d)[ & \frac{-\kappa-3}{r_{1}^{2}}+\frac{4 x_{1}^{2}}{r_{1}^{4}}-\frac{3 \kappa+1}{r_{2}^{2}} \\
& +\frac{4 \kappa x_{2}^{2}+(20-4 \kappa) c x_{2}-8 c^{2}}{r_{2}^{4}} \\
& \left.-\frac{32 c x_{2}^{3}-32 c^{2} x_{2}^{2}}{r_{2}^{6}}\right]
\end{aligned}
$$

$$
\begin{aligned}
S_{y x y}= & \frac{(-\kappa-3) x_{1}}{r_{1}^{2}}+\frac{4 x_{1}^{3}}{r_{1}^{4}}+\frac{2(\kappa-1) c-(3 \kappa+1) x_{2}}{r_{2}^{3}} \\
& +\frac{4 \kappa x_{2}^{3}+(28-4 \kappa) c x_{2}^{2}-24 c^{2} x_{2}}{2 r_{2}^{4}} \\
& +\frac{32 c^{2} x_{2}^{3}-32 c x_{2}^{4}}{r_{2}^{6}}
\end{aligned}
$$




$$
\begin{array}{rlrl}
D_{y x}= & (y-d)\left[\frac{2 x_{1}}{r_{1}^{2}}-\frac{2 \kappa\left(x_{2}-2 c\right)}{r_{2}^{2}}+\frac{8 c x_{2}\left(c-x_{2}\right)}{r_{2}^{4}}\right] & \text { where } \\
& +\left(\kappa^{2}-1\right) \theta_{2} & x_{1}=x-c & x_{2}=x+c \\
D_{y y}=-2 \kappa \log r_{1}-\frac{2 x_{1}^{2}}{r_{1}^{2}}-\left(\kappa^{2}+1\right) \log r_{2} & r_{1}=\sqrt{x_{1}^{2}+(y-d)^{2}} \\
+\frac{-2 \kappa x_{2}^{2}-4 c x_{2}+4 c^{2}}{r_{2}^{2}}+\frac{8 c x_{2}^{2}\left(x_{2}-c\right)}{r_{2}^{4}} & r_{2}=\sqrt{x_{2}^{2}+(y-d)^{2}} \\
& \theta_{2}=\arcsin \left(\frac{y-d}{r_{2}}\right)
\end{array}
$$

